2016-09-15

\title{
Spike-adding in parabolic bursters : The role of folded-saddle canards
}

\section{Desroches, M}

http://hdl.handle.net/10026.1/4835

10.1016/j.physd.2016.05.011

Physica D

All content in PEARL is protected by copyright law. Author manuscripts are made available in accordance with publisher policies. Please cite only the published version using the details provided on the item record or document. In the absence of an open licence (e.g. Creative Commons), permissions for further reuse of content should be sought from the publisher or author. 


\title{
Spike-adding in parabolic bursters: the role of folded-saddle canards
}

\author{
Mathieu Desroches ${ }^{\mathrm{a}}$, Martin Krupa ${ }^{\mathrm{a}}$, Serafim Rodrigues ${ }^{\mathrm{b}}$ \\ ${ }^{a}$ Inria Sophia Antipolis Méditerranée Research Centre, MathNeuro Team, \\ 2004 route des Lucioles - BP93, 06902 Sophia Antipolis, France \\ (mathieu.desroches@inria.fr,maciej.krupa@inria.fr) \\ ${ }^{b}$ School of Computing and Mathematics, Plymouth University, \\ Drake Circus, Plymouth PL4 $8 A A, U K$ \\ (serafim.rodrigues@plymouth.ac.uk)
}

\begin{abstract}
The present work develops a new approach to studying parabolic bursting, and also proposes a novel four-dimensional canonical and polynomial-based parabolic burster. In addition to this new polynomial system, we also consider the conductance-based model of the Aplysia R15 neuron known as the Plant model, and a reduction of this prototypical biophysical parabolic burster to three variables, including one phase variable, namely the Baer-Rinzel-Carillo (BRC) phase model. Revisiting these models from the perspective of slow-fast dynamics reveals that the number of spikes per burst may vary upon parameter changes, however the spike-adding process occurs in an explosive fashion that involves special solutions called canards. This spike-adding canard explosion phenomenon is analysed by using tools from geometric singular perturbation theory in tandem with numerical bifurcation techniques. We find that the bifurcation structure persists across all considered systems, that is, spikes within the burst are incremented via the crossing of an excitability threshold given by a particular type of canard orbit, namely the true canard of a folded-saddle singularity. However there can be a difference in the spike-adding transitions in parameter space from one case to another, according to whether the process is continuous or discontinuous, which depends upon the geometry of the folded-saddle canard. Using these findings, we construct a new polynomial approximation of the Plant model, which retains all the key elements for parabolic bursting, including the spike-adding transitions mediated by folded-saddle canards. Finally, we briefly investigate the presence of spike-adding via canards in planar phase models of parabolic bursting, namely the theta model by Ermentrout and Kopell.
\end{abstract}

Keywords: Parabolic bursting, spike-adding, folded-saddle canards

\section{Introduction}

Bursting oscillations are said to be parabolic when the burst initiates and terminates with arbitrary low frequency, hence exhibiting a parabolic-shaped curve when measuring the interspike intervals along a burst. In the time domain the observed oscillatory pattern consists of periodic bursts of activity "superimposed" onto a slow wave, which can be explained by the interaction between two 
oscillatory processes, one evolving on a slow timescale and the other evolving on a fast timescale. Mathematically, this bursting type translates to dynamical systems with at least four state variables, two slow variables and two fast variables. The dynamics of the slow and fast variables can be further reduced to phase variables resulting in either planar or three-dimensional models depending on whether the phase reduction is applied either to the fast processes or to both slow and fast processes; see the work of Bertram et al. [3], Golubitsky et al. [21], and Izhikevich [27, 28] for alternative approaches.

Parabolic bursting was first observed in Aplysia R15 ganglion cells and the first biophysical (conductance-based) model was proposed by Plant and colllaborators [44]. This model is presently known as the Plant model. This work inspired novel approaches based on numerical bifurcation analysis and singular perturbations, enabling a classification of bursting according to the properties of the underlying fast and slow dynamics. In [45, 47], Plant already compared the parabolic bursting behaviour of the full system and the behaviour of the fast system (obtained by freezing the slow dynamics). Subsequently, Rinzel and his collaborators $[1,48,49,56]$ applied the methods of singular perturbation theory to bursting and introduced the term "slow-fast dissection" to describe their approach. In particular, in [1] Baer, Rinzel and Carillo applied slow-fast dissection to a modified version of the Plant model and developed a threedimensional phase model of parabolic bursting which we will refer to as the Baer-Rinzel-Carillo (BRC) model. Ermentrout and Kopell [16] introduced an even more simplified model of parabolic bursting, involving two phase variables, known as Ermentrout-Kopell canonical model, a.k.a. theta or Atoll model, and studied its bifurcation structure; see also [57].

One of the main theoretical tools used to analyse slow-fast bursters, which we also employ here, is the dissection method [48, 50]. The idea is to freeze the dynamics of the slow variables $\mathbf{y}$ by setting $\varepsilon=0$ in the following "fasttime" parametrisation of a generic slow-fast system (with explicit timescale separation):

$$
\begin{aligned}
& \mathbf{x}^{\prime}=\mathbf{f}(\mathbf{x}, \mathbf{y}), \\
& \mathbf{y}^{\prime}=\varepsilon \mathbf{g}(\mathbf{x}, \mathbf{y}),
\end{aligned}
$$

where $0<\varepsilon \ll 1$ is a small parameter and where in the context of parabolic bursting $(\mathbf{x}, \mathbf{y}) \in \mathbb{R}^{2} \times \mathbb{R}^{2}$; see e.g. $[29,38,39]$ for general results on slowfast systems of the form (1). The slow variables are therefore considered as parameters entering the remaining differential equations for the fast variables $\mathbf{x}$; the limiting problem (for $\varepsilon=0$ ) obtained in this way is usually referred to as fast subsystem. In this setting, the bifurcation diagram of the fast subsystem with respect to the original two slow variables (seen as parameters), reveals the dynamical transitions between slow and fast phases of activity in the full fourdimensional system. Specifically, these transitions are between the quiescent phase, where the system tracks quasi-statically a branch of stable equilibria of the fast subsystem, and the burst (or active) phase, where the system follows a family of stable limit cycles of the fast subsystem.

A powerful graphical way to represent the slow-fast dissection method is to trace in the same phase-space projection a solution of the full system and the various bifurcation branches of the fast subsystem; see Fig. 1, which shows the 
result of this procedure in the Plant model. The periodic solution of the full $4 \mathrm{D}$ system (black) is superimposed onto the (green) surface $S^{0}$ of equilibria of the fast subsystem. This surface $S^{0}:=\{\mathbf{f}=\mathbf{0}\}$, associated with the $\varepsilon=0$ limit of the system, is also referred to as the critical manifold. It is folded along two curves, $F^{ \pm}$, that correspond to curves of saddle-node bifurcations of equilibria of the fast subsystem. The fold curves are equivalently defined by the algebraic condition $\operatorname{det}\left(\mathbf{f}_{\mathbf{x}}\right)=0$, where $\mathbf{f}_{\mathbf{x}}$ denotes the Jacobian of (1) with respect to the fast variables. In parabolic bursters like in any bursters, the fast oscillations constitutive of the burst are related to the presence of limit cycles in the fast subsystem. However, in the parabolic bursting case the fast subsystem has two parameters that correspond to the two slow variables of the full system. The family of limit cycles exists in a region of the corresponding two-parameter plane, and for each one-parameter slice of this region - obtained by fixing one parameter/slow variable and moving the other as a bifurcation parameter - the family terminates at a saddle-node on invariant circle (SNIC) bifurcation [28, $35,48]$. There is therefore a two-parameter family (a curve in the corresponding two-parameter plane) of SNIC bifurcation points in parabolic bursters. There could be other bifurcation scenarios, but this particular one is what corresponds to parabolic bursting oscillations, and we have verified this numerically for the models considered here. The curve of saddle-node bifurcations of equilibria associated with the SNIC bifurcations corresponds to a fold curve of the critical manifold $S^{0}$. In the case of the Plant model, the curve of SNIC correspond to the lower fold curve $F^{+}$of $S^{0}$. Note that the other upper fold curve $F^{-}$of $S^{0}$ is a regular fold curve, that is, it corresponds to saddle-node bifurcation points of the fast subsystem.

To date the investigations of parabolic bursting have mainly focused on the fast dynamics, and in particular on the consequences of the existence of a family of SNIC orbits. In this work we investigate in more detail the slow dynamics and the interaction between the slow and fast dynamics through the presence of a canard solution, a special solution that flows from an attracting to a repelling (locally) invariant manifold. We show that the number of spikes per burst is controlled by a so-called folded-saddle singularity, a special point located on the fold curve of the critical manifold. Defined in the singular limit $\varepsilon=0$, this point gives rise to a singular canard solution which for small $\varepsilon>0$ perturbs to the true canard $[2,58]$ (the term "true" refers to the existence of another special solution called the "faux canard", see below). In the context of parabolic bursting, we show that the spike-adding mechanism is entirely organised by the folded-saddle singularity and its associated canards. More precisely, the main contribution of this work is to show that for all 3D and 4D parabolic bursters that we consider, there exists a folded-saddle singularity and a homoclinic bifurcation occuring in the desingularised slow flow (for $\varepsilon=0$, see Section 3), that involves the folded saddle and its canard solutions. This bifurcation marks the transition (in the full system) from slow (subthreshold) wave activity to spiking solutions, and we call the corresponding event in the true slow flow a folded homoclinic bifurcation. Then, further spike-adding transitions all involve a passage through the true canard. We also highlight the fact that solution segments corresponding to the quiescent phase of these systems follow another special solution called the (singular) faux canard, which flows from a repelling sheet to an attracting sheet of $S^{0}$, in opposite direction to the singular true canard. The singular true canard and the singular faux canard correspond to the stable manifold 


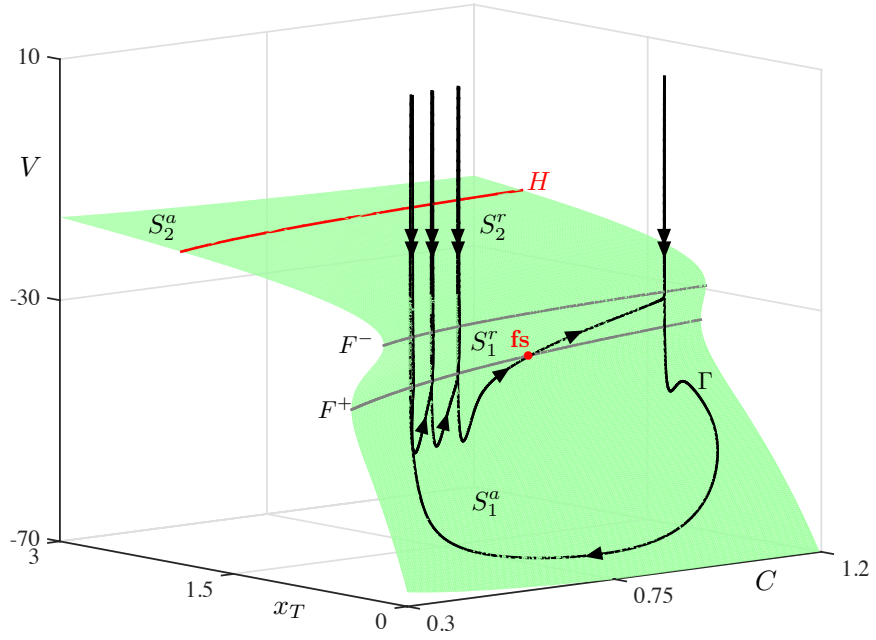

Figure 1: Bursting solution of the Plant model (1) containing a canard segment, represented in three-dimensional projection onto the $\left(x_{T}, C, V\right)$-space. The black orbit $\Gamma$ is a limit cycle with three fully developed spikes and one spike "almost" formed (close to the true canard). It follows both the attracting sheet $S_{1}^{a}$ and the repelling sheet $S_{1}^{r}$ of the critical manifold $S^{0}$ (green surface), whose fold curves $F^{ \pm}$separate the external sheets $\left(S_{1}^{a}\right.$ and $\left.S_{2}^{r}\right)$ from the repelling sheet $S_{1}^{r}$. The underlying folded saddle is shown by a red dot and labelled fs. There is also a curve of Hopf bifurcation points $H$ (red curve) of the fast subsystem. The arrows along $\Gamma$ indicate the direction of the flow.

and to the unstable of the saddle equilibrium of the desingularised slow flow, respectively; see Section 3.1. We propose a new polynomial model of parabolic bursting that retains all salient features of the Plant model, in particular the canard-induced spike-adding transitions. This model is built as an extension of a modified version of the Hindmarsh-Rose burster $[11,26]$.

The theta model by Ermentrout and Kopell [16] is too simple to have a folded saddle. We include an analysis of this model for completeness of our study, showing that spike-adding arises by means of jump-on canards, that is, solutions flowing along a repelling (locally) invariant manifold after having completed a fast segment $[8,13]$. We point out that recent studies of slow-fast systems on $\mathbb{T}_{1}$ and $\mathbb{T}_{2}[23,53,54,55]$ provide proofs of the existence and genericity of canards in this context. These results are applicable to the Ermentrout-Kopell theta model and, therefore, to a large class of phenomenological parabolic bursters.

The rest of the paper is organised as follows. In Section 2, we give initial numerical evidence of the canard-induced spike-adding structure in the Plant model. In Section 3, we present a novel polynomial model, based on a modified Hindmarsh-Rose system, which has all the dynamical features of the Plant model. All calculations showing the presence of a folded saddle are carried out with this canonical model. In Section 4, we consider the BRC model and show that it also possesses a folded-saddle singularity. However, we find that this phase model does not entirely reproduce the same spike-adding mechanism as in the Plant model, the reason being the geometry of the critical manifold and, therefore, of the true canard. Our analysis shows that four dimensions 
are needed to fully reproduce parabolic bursting as found in the Plant model. Finally, in Section 5 we briefly analyse the presence of canards in Atoll models, and we relate them to recent results about canards on the torus.

\section{Plant biophysical model of the Aplysia R15 neuron}

We begin our study of spike-adding in parabolic bursters with the Plant model for the Aplysia R15 bursting neuron, which was introduced in 1978 [46], later simplified in 1981 [47], and ever since considered as a classical conductancebased parabolic burster. The final version is a five-dimensional model given as follows:

$$
\begin{aligned}
\dot{V} & =\left(g_{I} s_{I}^{3}(V) y_{I}+g_{T} x_{T}\right)\left(V_{I}-V\right)+\left(g_{K} x_{K}^{4}+\frac{g_{p} c}{K_{p}+c}\right)\left(V_{K}-V\right)+g_{L}\left(V_{L}-V\right) \\
& =f\left(V, x_{T}, x_{K}, y_{I}, c\right), \\
\dot{x_{i}} & =\left(s_{i}(V)-x_{i}\right) / \tau_{x_{i}}, \quad i=T, K \\
\dot{y_{I}} & =\left(z_{I}(V)-y_{I}\right) / \tau_{y_{I}}, \\
\dot{c} & =\rho\left(K_{c} x_{T}(V)\left(V_{C a}-V\right)-c\right) ;
\end{aligned}
$$

see [47] for details on the sigmoid-shaped activation functions and classical parameter values. The preliminary studies by Plant in [47] suggested that there is an underlying slow sub-system of model (2). However, given that the model is based on the Hodgkin-Huxley formalism, it was not clear how to identify an obvious time-scale separation (i.e. a small parameter $\varepsilon$ ). In [49], Rinzel introduced the time scale separation based on a direct simulation of (2) with $x_{T}$ and $c$ identified as slow and the remaining three variables identified as fast. Rinzel understood the shape of parabolic bursting by analyzing the bifurcation structure of the fast system. Specifically, it becomes clear that the fast system has a curve of SNIC points that depends on the two parameters obtained by freezing the remaining two slow variables. Following this approach, we can formally recast system (2) as a slow-fast system, the timescale separation parameter $\varepsilon$ being equal to $\min \left(1 / \tau_{x_{T}}, \rho\right)$. Moreover, by virtue of the well-established singular perturbation theory we can now further analyze the slow-fast structure of the problem when $\varepsilon$ tends to zero. Doing so in the (equivalent) slow-time parametrisation of system (2) yields the critical manifold $S^{0}$, which is given by the following set of algebraic equations:

$$
\begin{aligned}
f\left(V, x_{T}, x_{K}, y_{I}, c\right) & =0, \\
x_{K} & =s_{K}(V), \\
y_{I} & =z_{I}(V) .
\end{aligned}
$$

A visualisation of the corresponding surface (shown in green in Fig. 1) confirms that it has locally two fold curves $F^{ \pm}$. Besides, for specific values of parameter $G_{I}$, we find bursting solutions that do not always jump at the fold (SNIC) curve $F^{+}$, but rather follow the repelling sheet $S_{1}^{r}$ of the critical manifold $S^{0}$ in between the two fold curves. After completing this repelling segment, which we can already interpret as a canard segment, the trajectory makes a final spike before returning to quiescence. This figure clearly shows that the fourth 
spike of this bursting cycle is different in nature from the other three, in that it corresponds to a jump from $F^{-}$as opposed to a jump from $F^{+}$for the first three spikes. This suggests that, in order to "add" one more spike upon parameter variation, system (1) must possess a family of limit cycles with canard segments that extend from one fold curve to the other. This behaviour is reminiscent of canard-induced spike-adding transitions found in square-wave bursters [11] and suggests to find the signature of canard dynamics in this parabolic bursting context. Given that there are two slow variables, we look for folded singularities, that is special points located on a fold curve of the critical manifold and near which the flow of the full system continues past the fold to follow the repelling part of $S^{0}$ instead of jumping, hence producing a canard segment (see e.g. [9] for details). We can find a folded-saddle singularity fs in this system, as will become clear in subsequent sections. Note that there is a second repelling part on $S^{0}$, which we denote by $S_{2}^{r}$, on the other side of the upper fold curve $F^{-}$. This is due to the presence of a curve of Hopf bifurcation points of the fast subsystem on the upper sheet of $S^{0}$, which divides this sheet into an attracting part $S_{2}^{a}$ and a repelling part $S_{2}^{r}$. This is typical of parabolic bursting and explains the actual burst, which is due to the presence of a two-parameter family of limit cycles in the fast subsystem.

Since the Plant model is biophysical, its mathematical structure is rather complex and makes it difficult to carry out a lot of analytical calculations with it. This motivates us to construct a polynomial model that retains all the dynamics of the Plant model while being amenable to analysis. Our model has the minimal number of slow and fast variables necessary to capture all the features of parabolic bursting. In particular, it has the correct slow flow, that is, the limit when $\varepsilon \rightarrow 0$ of the slow dynamics, which reveals the presence of a folded-saddle singularity.

\section{A canonical model with two slow variables and two fast variables}

Given the complexity of the Plant model, that is, its large dimension and strong nonlinearities, a natural question is then whether one can construct a canonical model of the spike-adding mechanism in such parabolic bursters. Reduced models have already been constructed; an example which quite accurately reproduces the dynamics of the Plant model is the BRC model, a system with two slow variables and one fast phase variable [1], see section 4 . In this section we develop a $4 \mathrm{D}$ model, with two slow variables and two fast variables, which is a variation of the Hindmarsh-Rose model [26], adapted from our earlier paper [11]. Our model has more details in the fast dynamics and therefore, as we argue below, reproduces additional details of the dynamics of the Plant model. The equations are as follows:

$$
\begin{aligned}
& x^{\prime}=c\left(x-x^{3} / 3-y+z+I\right), \\
& y^{\prime}=\left(x^{2}+d x-b y+a\right) / c, \\
& z^{\prime}=\varepsilon\left(z-s\left(x-x_{1}\right)\right), \\
& I^{\prime}=\varepsilon\left(k-h_{x}\left(x-x_{\text {fold }}\right)^{2}-h_{y}\left(y-y_{\text {fold }}\right)^{2}-h_{I}\left(I-I_{\text {fold }}\right)\right),
\end{aligned}
$$

where $I$ is the applied current that evolves slowly. The values of the parameters $a, b, c, d, x_{1}$ and $s$ are fixed at $a=0.08, b=0.71, c=3, d=1.8, s=4$, 
$x_{1}=-1.6$ and the singular parameter $\varepsilon$ has the value $\varepsilon=0.0004$. The role and the settings of the parameters $x_{\text {fold }}, y_{\text {fold }}$ and $I_{\text {fold }}$ will be explained below. System (4) has a SNIC bifurcation in the fast subsystem, which is one of the key features of parabolic bursting and also occurs in the BRC model of [1]. In addition (4) has an $S$ shaped critical manifold which allows for continuous spike adding transitions. Recall that the critical manifold is defined as the fast nullsurface $\left\{x^{\prime}=0\right\} \cap\left\{y^{\prime}=0\right\}$ for $\varepsilon=0$.

We feel that the main contribution of this work is to investigate in more detail the slow dynamics associated with parabolic bursting. We will first carry out such a study in the context of (4) and subsequently show that both the Plant model and the BRC model model have the same features from the viewpoint of canard dynamics, even though the spike-adding transitions occur along continuous branches in parameter space in the former and along discontinuous branches in the latter. We will also pay some attention to the fast dynamics, showing that our system reproduces all the salient features of the fast dynamics of the Plant model.

\subsection{Slow dynamics of (4)}

As mentioned above, the focus of this work is on the slow flow. We will now describe the features of the slow flow of (4). Our assertions are mainly based on numerical exploration. Proving these results analytically remains an open problem. We begin by rewriting (4) in the "slow time" formulation:

$$
\begin{aligned}
\varepsilon \dot{x} & =c\left(x-x^{3} / 3-y+z+I\right), \\
\varepsilon \dot{y} & =\left(x^{2}+d x-b y+a\right) / c, \\
\dot{z} & =z-s\left(x-x_{1}\right), \\
\dot{I} & =k-h_{x}\left(x-x_{\text {fold }}\right)^{2}-h_{y}\left(y-y_{\text {fold }}\right)^{2}-h_{I}\left(I-I_{\text {fold }}\right) .
\end{aligned}
$$

The time variable in (5) is given by $t=\varepsilon \tau$, where $\tau$ is the time parametrisation of system (4). By setting $\varepsilon=0$ we obtain the reduced problem or slow subsystem, a system of algebro-differential equations whose dynamics approximate the slow flow. The two algebraic constraints define the critical manifold $S^{0}$, which is the phase space of the reduced problem. It is easy to see that $S^{0}$ is defined by the equations:

$$
\begin{aligned}
& y=\frac{1}{b}\left(x^{2}+d x+a\right) \\
& z=\frac{x^{3}}{3}+\frac{x^{2}+d x+a}{b}-x-I .
\end{aligned}
$$

and is an S-shaped surface with two folds $F^{ \pm}$defined by $\partial z / \partial x=0(z$ given by (7)), that is,

$$
x_{\mathrm{fold} \pm}=-\frac{1}{b}(1 \pm \sqrt{1+b(b-d)}) .
$$

In particular $x_{\text {fold }}$ appearing in (4) is $x_{\text {fold }+}$ and $y_{\text {fold }}$ is the corresponding $y$ value. The value $I_{\text {fold }}$ is chosen so that the slow nullcline of the modified HR burster $\left\{z=s\left(x-x_{1}\right)\right\}$ passes exactly through $F^{-}$, that is

$$
I_{\mathrm{fold}} \pm=\frac{x_{\mathrm{fold}}^{3}}{3}+\frac{x_{\mathrm{fold}}^{2}+d x_{\mathrm{fold}}+a}{b}-x_{\mathrm{fold}}-s\left(x_{\mathrm{fold}}-x_{1}\right) .
$$


Note that the fold lines divide $S^{0}$ into three sheets and the reduced problem restricted to each of these sheets is non-singular. We focus on the lower

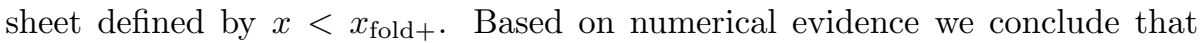
the reduced system restricted to the lower sheet has the following sequence of dynamics, upon variation of the parameter $k$ :

1. Stable equilibrium;

2. Hopf bifurcation leading to a stable limit cycle which attracts the singular faux canard;

3. An event, which we choose to term folded homoclinic bifurcation - and which corresponds to a true homoclinic bifurcation in the desingularised reduced system, see below - characterised by a collision of the limit cycle with the fold line $F^{+}$, leading to the disappearance of the limit cycle; The singular periodic orbit is a connection from the singular faux canard to the singular true canard, passing through a folded-saddle singularity.

4. Unstable equilibrium with all non-equilibrium trajectories eventually terminating on the fold line $F^{+}$.

Note that due to the singular character of the reduced problem, the orbit connecting the folded saddle to itself has finite passage time. In the desingularised version of the reduced system, which we discuss below, the time parametrisation is changed so that the folded saddle point becomes a saddle type equilibrium and this connecting orbit becomes a true homoclinic connection. Therefore, we refer to this singular periodic orbit as a folded homoclinic orbit and the associated global bifurcation (in the desingularised version of the reduced system) as a folded homoclinic bifurcation. In the sequel we will show that the folded homoclinic bifurcation corresponds to a well known homoclinic bifurcation in the desingularised reduced system (DRS), which we introduce below. We will argue that the folded homoclinic bifurcation is the organising center of the transition between the slow periodic dynamics and parabolic bursting.

We now introduce the desingularised reduced system (DRS). Note that algebraic condition (7) gives an expression of $z$ as a function of $x$ and $I$. Differentiating (7) with respect to time and using (5) we obtain a differential equation for $x$

$$
\begin{aligned}
\left(x^{2}+\frac{2}{b} x+\frac{d}{b}-1\right) \dot{x}= & \dot{z}+\dot{I} \\
= & \frac{x^{3}}{3}+\frac{x^{2}+d x+a}{b}-x-I-s\left(x-x_{1}\right)+ \\
& k-h_{x}\left(x-x_{\text {fold }}\right)^{2}-h_{y}\left(y-y_{\text {fold }}\right)^{2}- \\
& h_{I}\left(I-I_{\text {fold }}\right) .
\end{aligned}
$$

We can now express the reduced system in terms of the variables $x$ and $I$, using (10) and the constraints (6) and (7). This procedure gives the following formulation of the reduced system:

$$
\begin{aligned}
\left(x^{2}+\frac{2}{b} x+\frac{d}{b}-1\right) \dot{x}= & \frac{x^{3}}{3}+\frac{x^{2}+d x+a}{b}-x-I-s\left(x-x_{1}\right)+k- \\
& h_{x}\left(x-x_{\text {fold }}\right)^{2}-h_{y}\left(y-y_{\text {fold }}\right)^{2}-h_{I}\left(I-I_{\text {fold }}\right) \\
\dot{I}= & k-h_{x}\left(x-x_{\text {fold }}\right)^{2}-h_{y}\left(y-y_{\text {fold }}\right)^{2}-h_{I}\left(I-I_{\text {fold }}\right) .
\end{aligned}
$$



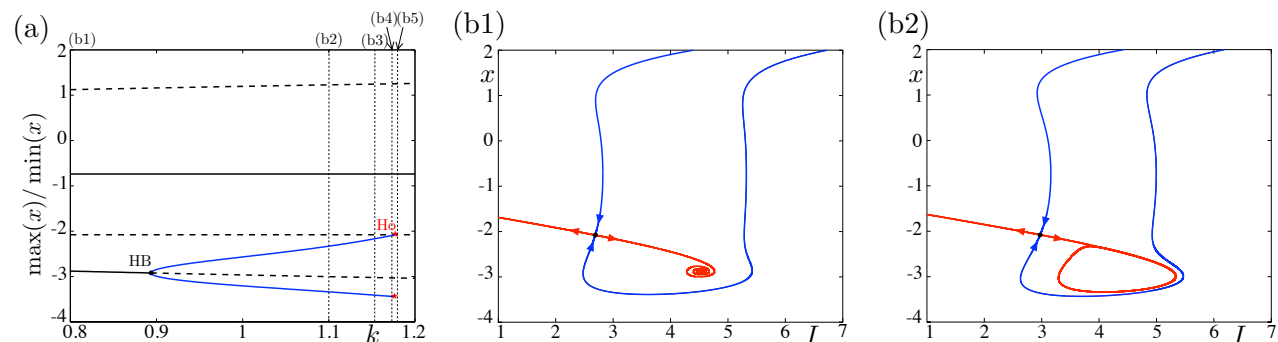

(b3)
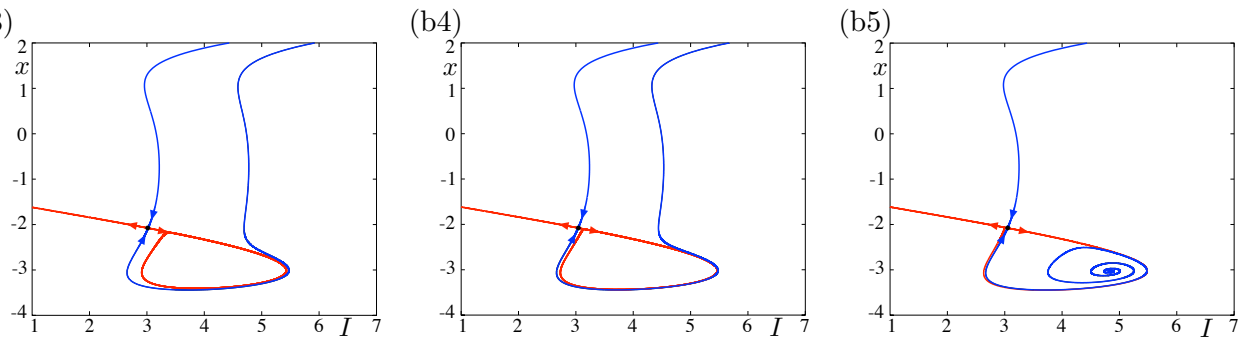

Figure 2: Main dynamical elements of the slow flow of the canonical parabolic bursting model (4). Panel (a): Bifurcation structure of the DRS with respect to parameter $k$. Panels (b1)-(b5): phase portrait of the DRS for a few selected values of $k$ across the bifurcation diagram shown in panel (a).

The resulting system is formulated in terms of $x$ and $I$ only, and the constraints (6) and (7) have been eliminated. Note that (11) is singular along the fold set $F=F^{+} \cup F^{-}$, which reflects that singularity of $S^{0}$. In order to extend the domain of definition of (11) up to the fold set $F$, one typically desingularises it by performing a singular time rescaling, here by a factor $x^{2}+\frac{2}{b} x+\frac{d}{b}-1$; see for instance $[5,9]$ for the general theory. Therefore, one obtains a planar system given by

$$
\begin{aligned}
\dot{x}= & \frac{x^{3}}{3}+\frac{x^{2}+d x+a}{b}-x-I-s\left(x-x_{1}\right)+k-h_{x}\left(x-x_{\text {fold }}\right)^{2}- \\
& h_{y}\left(y-y_{\text {fold }}\right)^{2}-h_{I}\left(I-I_{\text {fold }}\right) \\
\dot{I}= & \left(x^{2}+\frac{2}{b} x+\frac{d}{b}-1\right)\left(k-h_{x}\left(x-x_{\text {fold }}\right)^{2}-h_{y}\left(y-y_{\text {fold }}\right)^{2}-h_{I}\left(I-I_{\text {fold }}\right)\right)
\end{aligned}
$$

which is regular everywhere. System (12) is called the Desingularised Reduced System (DRS). Its dynamics away from the fold lines differs from the dynamics of the reduced system by time parametrisation only, yet (12) is easier to study both theoretically and numerically as it has no singularities. In particular, equilibria of (12) lying on $F$ correspond to points for which the dynamics of (11) is tangent to $F$ and where the solutions of (11) can sometimes be continued across $F$. Such points are called pseudo-equilibria or folded singularities. As we show below there exists a unique folded saddle located on $F^{+}$. In addition, our numerical results show that (12) has the following sequence of dynamics, upon variation of the parameter $k$ :

1. Stable equilibrium;

2. Hopf bifurcation leading to a stable limit cycle;

3. Homoclinic bifurcation through which the stable limit cycle disappears.

This is precisely the bifurcation sequence whose existence we establish numerically and which translates to the bifurcation sequence of the reduced system 
described earlier in this section. The relevant orbits are shown in Figure 2.

To establish the existence of the folded saddle we focus on the folded singularity satisfying $x_{\mathrm{f}}<0$, that is,

$$
x_{\mathrm{f}}=-\frac{1}{b}(1+\sqrt{1+b(b-d)})=x_{\text {fold }+} .
$$

From the previous equality it follows immediately that $y_{\mathrm{f}}=y_{\mathrm{fold}+}$. Using the $x$-equation of the DRS, we find that

$$
I_{\mathrm{f}}=\frac{x_{\mathrm{f}}^{3} / 3+\left(x_{\mathrm{f}}^{2}+d x_{\mathrm{f}}+a\right) / b-x_{\mathrm{f}}-s\left(x_{\mathrm{f}}-x_{1}\right)+k+h_{I} I_{\mathrm{fold}+}}{1+h_{I}} .
$$

In the first four terms of the numerator above, we recognise the expression of $I_{\text {fold }+}$ given in (9). Therefore, we have

$$
I_{\mathrm{f}}=I_{\mathrm{fold}+}+\frac{k}{1+h_{I}} .
$$

Now, the Jacobian matrix of the DRS at the folded singularity $\left(x_{\mathrm{f}}, I_{\mathrm{f}}\right)$ is given by

$$
\mathrm{J}=\left(\begin{array}{cc}
-s & -\left(1+h_{I}\right) \\
2\left(x_{\mathrm{f}}+\frac{1}{b}\right)\left(k-h_{I}\left(I_{\mathrm{f}}-I_{\text {fold }}\right)\right) & 0
\end{array}\right) .
$$

Using (14) and (15), we see that the lower left entry further simplifies to

$$
2\left(x_{\mathrm{f}}+\frac{1}{b}\right) \frac{k}{1+h_{I}} .
$$

Consequently, the condition to have a saddle (strictly negative determinant) is that $x_{\mathrm{f}}<-1 / b$ ( $k$ has been chosen strictly positive), that is,

$$
b(b-d)>-1 .
$$

Furthermore, one can compute the saddle quantity $\sigma^{*}$ [28], evaluated at the folded sadde point $\left(x_{\mathrm{f}}, I_{\mathrm{f}}\right)$ and defined as the sum of the eigenvalues of the folded saddle viewed as a saddle equilibrium of the DRS. Therefore, $\sigma^{*}$ is the trace of $\mathrm{J}$, hence it is equal to $-s<0$. One can then conclude that the homoclinic bifurcation involving the folded saddle is supercritical and that the family of limit cycles terminating at this bifurcation is stable.

\subsection{Fast dynamics}

The fast subsystem of (4) is a planar system with two main parameters corresponding to the slow variables of the full dynamics, namely, $z$ and $I$. The set of equilibria of (4) with $\varepsilon=0$ is equal to the critical manifold $S^{0}$, given by (6)-(7), see Figs. 3, 4. The lower sheet of $S^{0}$ is stable for the fast dynamics, hence this is the region where the quiescent phases of the dynamics of the full system takes place. The middle sheet of $S^{0}$ is of saddle type. At the upper fold the stability changes to fully unstable, originally of node type, later changing to focus and finally becoming stable along a line of Hopf bifurcations, see Figs. 3, 4. The Hopf bifurcation gives rise to a region of oscillatory dynamics of the fast subsystem ending at a SNIC bifurcation, with the SNIC orbits corresponding to a connection from the lower fold to itself. Such orbits provide the fast part 
(a1)

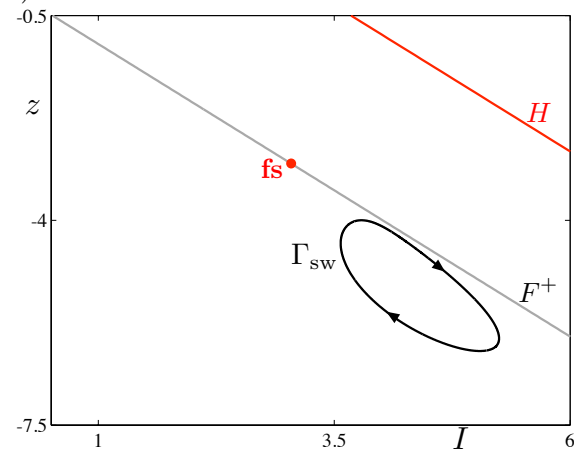

(a2)

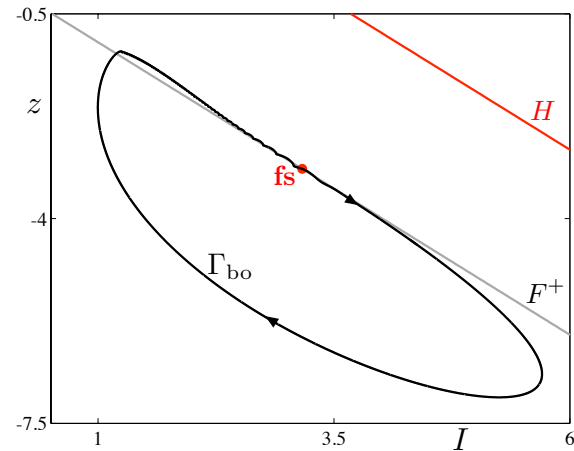

(b1)

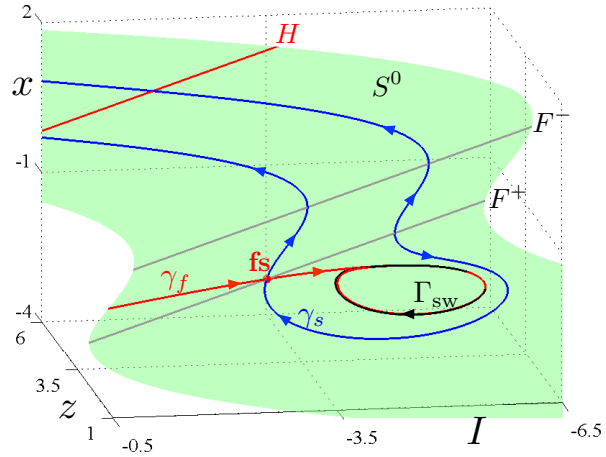

(b2)

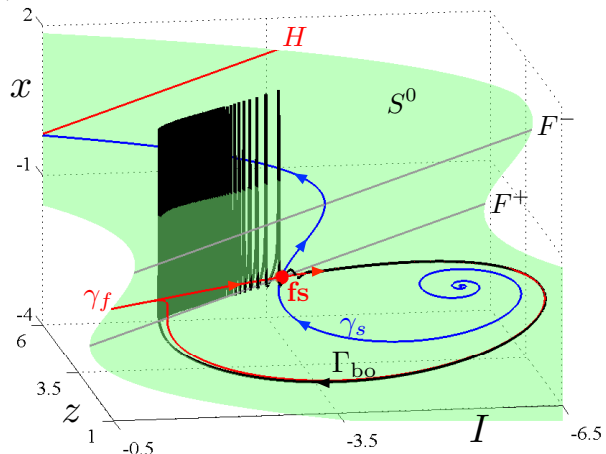

Figure 3: Transition from slow wave $\Gamma_{\mathrm{sw}}$ (top panels) to bursting $\Gamma_{\text {bo }}$ (bottom panels) solutions of the canonical parabolic bursting system (4). Each (a) panel shows a projection of the limit cycle onto the plane of slow variables $(z, I)$, together with the curve of SNIC points $F^{+}$ (fold curve of the critical manifold $S^{0}$ ) and the curve $H$ of Hopf points of the fast subsystem. In this projection, the slow wave $\Gamma_{\mathrm{sw}}$ stays entirely in the half-plane to the left of the SNIC curve, that is, it corresponds to pure quiescence. On the other hand, a bursting orbit like $\Gamma_{\text {bo }}$ crosses the SNIC curve twice, at the beginning and at the end of the burst. The (b) panels show a 3D projection, in the $(x, z, I)$ phase space, where we also show the cubic-shaped critical manifold $S^{0}$, the folded saddle fs, the fold curves $F^{ \pm}$, the singular true canard $\gamma_{s}$ and the singular faux canard $\gamma_{f}$. Parameter values: $k \approx 1.05$ in (a1)-(b1), $k=1.3$ in (a2)-(b2). In all panels, the arrows indicate the direction of the flow.

of the mechanism of parabolic bursting [48].These features of the fast dynamics are well-known in parabolic bursters and are also present in the Plant model. In the next section we combine them with the knowledge of the slow flow, as described in Section 3.1, obtaining a more complete picture of the geometry of bursting solutions and the spike-adding transitions.

\subsection{Dynamics of the full system}

In this section we roughly describe the dynamics of parabolic bursting, using heuristic arguments based on geometric singular perturbation theory, without providing rigorous proofs. Our arguments are based on the information on the slow flow (Section 3.2) and the fast flow (Section 3.1). Combining the information from both sources, we can get a better understanding of the shape of bursting cycles of the full system — shown in black in Fig. 3(b2) — as well 

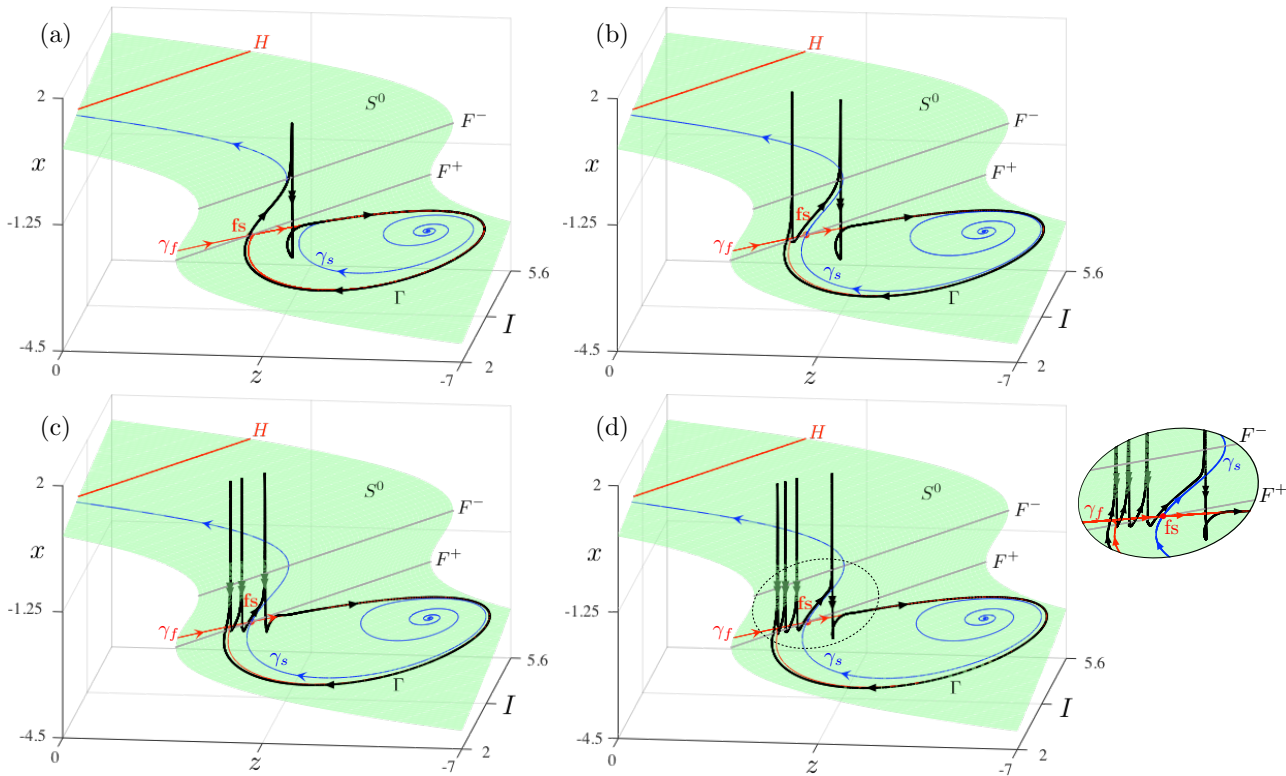

Figure 4: Solutions of the canonical parabolic bursting system (4) containing canard segments during the adding of the first, second, third and fourth spike in panels (a), (b), (c) and (d), respectively. In each panel, the black orbit $\Gamma$ is a limit cycle with one fully developed spike and one spike "almost" formed (close to maximal canard). It follows both attracting and repelling parts of the critical manifold (green surface). The blue curve is the singular true canard $\gamma_{s}$, corresponding to the stable manifold of the folded saddle (red dot labelled fs) seen as a saddle equilibrium of the DRS; the red curve is the singular faux canard $\gamma_{f}$. The inset next to panel (d) shows a zoom near the folded saddle as the fourth spike is being created. In all panels, the arrows indicate the direction of the flow.

as the geometric mechanism underpinning the spike-adding process for these cycles - shown in Fig. 4, for four nearby values of parameter $k$. Indeed, the alternation between bursting phases and quiescent phases corresponds to the crossing of lower SNIC curve $F^{+}$(see Figure $3(\mathrm{a} 2)$ ), and this is characteristic of parabolic bursters. Parabolic bursting orbits all start and end their burst phase by flowing near $F^{+}$except for orbits containing canard segments, when a new spike is being added. Our more detailed analysis of the slow flow (Section 3.1) reveals novel features of the dynamics, mainly due to the true canard that exists near the folded saddle. The family of faux canards existing by a result of [58] (see also [41]), also plays an important role. We fix two choices of Fenichel slow manifolds $S^{r, \varepsilon}$ and $S^{a, \varepsilon}$, that extend to the neighbourhood of the folded saddle. It is well known that for a fixed choice of $S^{a, \varepsilon}$ and $S^{r, \varepsilon}$ there exists a unique true canard solution which originates in $S^{a, \varepsilon}$, passes through a neighborhood of the folded saddle and connects to $S^{r, \varepsilon}[58]$.

Recall the three parameter regions of the dynamics of (11): the region characterised by the existence of a stable equilibrium, the region characterised by the existence of a stable periodic orbit, which in particular attracts the singular faux canard, and the region where the singular faux canard connects to the fold line. The last two regions mentioned above are separated by the connection from the singular faux canard to the singular true canard, which we term folded homoclinic bifurcation (see Section 3.1). Similarly, for $\varepsilon>0$, there exists a 
region of quiescent behaviour, where (4) has either an equilibrium or a stable periodic orbit entirely contained in $S^{a, \varepsilon}$ (see Figure 3 (a1-b1)), and the bursting region (see Figure 3 (a2-b2)). The transition from quiescence to bursting occurs as the quiescent periodic orbit approaches the true canard and starts developing a canard segment. As the parameter is varied ( $k$ in our case) the periodic orbit follows the true canard closer and closer, eventually including a maximal canard segment and jumping off the top fold, see Fig. 4(a).

Note that the dynamics on $S^{a, \varepsilon}$ in a small but $O(1)$ neighborhood of the folded saddle has the properties of the dynamics near a saddle point, which implies that the flow is strongly contracting. In other words, all trajectories that start close to the folded saddle and remain on $S^{a, \varepsilon}$ for a sufficiently long time are attracted to each other, forming a thin tube, $O(\varepsilon)$ close to the singular faux canard. Regular bursting corresponds to the case when the trajectories in this tube reach the vicinity of a jump point on the fold on the opposite side of the true canard and subsequently follow the fast dynamics. An orbit segment following the SNIC dynamics returns to the vicinity of the fold. It passes within $O\left(\varepsilon^{2 / 3}\right)$ from the fold line after having made approximately a full rotation along the fast connecting orbit [32]. As shown in Figure 3 (a2-b2), the orbit must finally return to the fold region and enter the contracting tube, which means that it re-enters the quiescent phase. Other instances of spike adding correspond to the cases when the returning trajectory from a fast oscillation following the SNIC orbit connects to the true canard, see Figure 4 (b-d) and occur according to the same mechanism as the creation of the first burst.

A faux canard may facilitate the explanation of this phenomenon. Szmolyan and Wechselberger [58, Proposition 4.3] prove that there exists an open set in $\mathbb{R}^{4}$ which contains open subsets of both $S^{a, \varepsilon}$ and $S^{r, \varepsilon}$ and consists of trajectories that first follow $S^{r, \varepsilon}$ and then $S^{a, \varepsilon}$. This result does not imply that there are any faux canards that continue from $S^{r, \varepsilon}$ to $S^{a, \varepsilon}$, but it is possible to modify $S^{a, \varepsilon}$ so that such faux canards exist. We assume henceforth that $S^{a, \varepsilon}$ has this property. Then the thin tube mentioned above can be defined as centered at the faux canard, which now (transiently on $O(\varepsilon)$ scale) attracts the dynamics coming from the fold. The transition to bursting can be roughly described as a connection from the faux canard to the true canard in the sense that, for parameter values past this event, the attractor of the system is not a slow wave anymore but a bursting orbit ; see Fig. 3(b1-b2).

Faux canards have recently been studied by Mitry and Wechselberger [41] who discovered that they can lead to rotational behaviour of the trajectories. Since the trajectories returning from a parabolic burst must pass close to the family of faux canards, some of this oscillatory behaviour may be a part of parabolic bursting; see e.g. Fig. 3(b2) for numerical evidence of this point.

Numerical exploration with AUTO [14] shows a continuous transition upon variation of $k$ from the region of slow oscillations to the region of parabolic bursting with many spikes; see Fig. 6 (in the cases of the Plant and the BRC models, respectively). We believe that the entire sequence of bifurcations is very intricate and it would be a challenging problem to obtain its full picture. A more in-depth study of this system will be a subject of future work.

\subsection{Back to the Plant model}

The previous sections indicate that the dynamics of our prototypical parabolic burster (4) captures all the essentials of the Plant model's behaviour, with also 
(a)

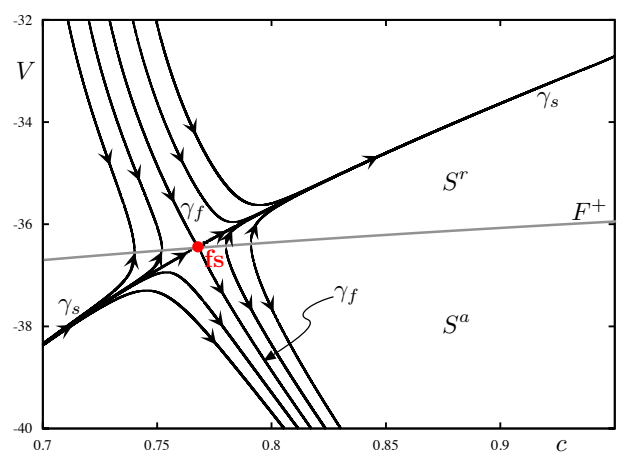

(b)

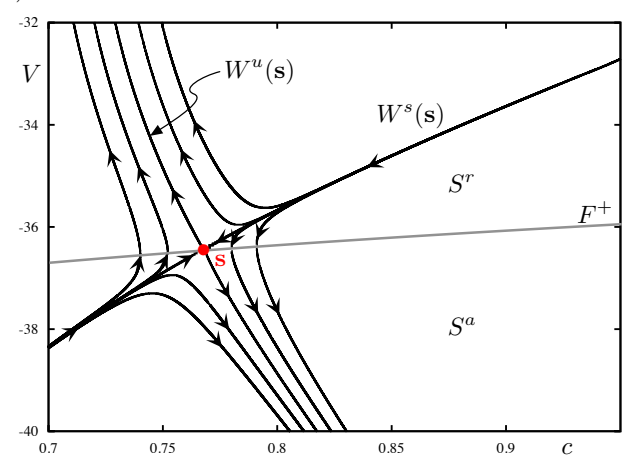

Figure 5: (a) Slow flow of the Plant model (1), computed by solving the associated DifferentialAlgebraic Equation (17) (DAE). (b) Flow of the Desingularized Reduced System (DRS) for the Plant model, showing the characteristics of a saddle equilibrium $\mathbf{s}$ with its stable manifold $W^{s}(\mathbf{s})$ and its unstable manifold $W^{u}(\mathbf{s})$. In the "true" reduced flow, it implies that this point is a folded saddle fs and the two special trajectories are the singular true canard $\gamma_{s}$ and the singular faux canard $\gamma_{f}$.

the same geometrical configuration in the (three-dimensional projection of the) phase space. Namely, a cubic-shaped critical manifold with two fold curves, the lower one corresponding to a SNIC curve of the fast subsystem, and a folded-saddle singularity on the lower fold curve organising the spike-adding mechanism. Analytical calculation with a high-dimensional conductance-based model such as the Plant model are tedious, this is why we only give numerical evidence of this claim. For the parameter values corresponding to Fig. 1, which are in the range considered by Plant and Baer, Rinzel and Carillo, the so-called normal switching conditions [39] are not satisfied; that is,

$$
\left(f_{x_{T}} \dot{x}_{T}+f_{c} \dot{c}\right)_{\left.\right|_{\mathrm{fs}}}=0,
$$

where $f_{x}$ denotes the partial derivative of $f$ with respect to variable $x$. This is characteristic of folded singularities [58]. This algebraic calculation is messy for system (1) due to its strong nonlinearities (sigmoid-type functions) and high dimension. We have performed these calculations with the software MATHEMATICA [36], however we refrain from showing the rather lengthy expressions that we obtained. The evaluation of formula (16) at the numerically computed point, fs, indeed gives zero. To confirm these symbolic computations, we provide numerical evidence of the presence of a folded saddle in system (1) by computing the flow of the reduced system

$$
\begin{aligned}
f\left(V, x_{T}, x_{K}, y_{I}, c\right) & =0, \\
x_{K} & =s_{K}(V), \\
y_{I} & =z_{I}(V), \\
\dot{x_{T}} & =k\left(s_{i}(V)-x_{i}\right), \\
\dot{c} & =K_{c} x_{T}(V)\left(V_{C a}-V\right)-c,
\end{aligned}
$$

where we rewrite $1 / \tau_{x_{T}}$ as $\rho k$. Fixing the ratio between $\rho$ and $\tau_{x_{T}}$ enables us to consider that system (1) has two slow variables and to derive the DifferentialAlgebraic Equation (17) (DAE) which governs the reduced system of the Plant 
model. The associated slow flow takes place on the critical manifold $S^{0}$ and approximates the slow dynamics of system (1) in the singular limit $\varepsilon=0$. System (17) can be simulated using adequate numerical schemes for DAEs and we obtain the computed reduced slow flow presented in Fig. 5 panel (a); the computation was performed using the DAE solver implemented within the software package XPPAUT [17]. This phase portrait is typical of a folded-saddle singularity (indicated by a red dot). Indeed, by reversing the time orientation on the repelling sheet $S^{r}$ of the critical manifold - that is, rescaling time by a factor - $f_{V}$, which is negative on the repelling sheet $S^{r}$ - we obtain the phase portrait shown in panel (b), which possesses a saddle equilibrium $\mathbf{s}$ with its stable manifold $W^{s}(\mathbf{s})$ and its unstable manifold $W^{u}(\mathbf{s})$. This corresponds to the DRS of the Plant model. We conclude that the slow flow of Plant model indeed has a folded saddle, and that two associated singular canards exist, corresponding to the special trajectories $W^{s, u}(\mathbf{s})$ of the DRS: the true canard $\gamma_{s}$ and the faux canard $\gamma_{f}$.

Furthermore, the full system's bifurcation diagram in parameter $G_{I}$ displays the structure of multiple explosive transitions that correspond to each spikeadding event; see Fig. 6. Back to Fig. 1, the limit cycle $\Gamma$ illustrates well the effect of the folded-saddle singularity fs on the burst part of this periodic solution. The singular true canard perturbs to a trajectory of the full system for $0<\varepsilon \ll 1$, which in this context is part of a limit cycle lying on the first quasi-vertical segment of the branch shown in Fig. 6 (a). The addition of a spike, upon parameter variation, therefore corresponds to the crossing of the true canard, which for this case corresponds to the excitability threshold. That role of excitability threshold for the true canard associated with a folded saddle has already been analysed in [40] in a different model, and it is directly related to the role of stable manifolds of saddle equilibria as repelling slow manifolds in planar excitable models [10, 28].

This canard transition between bursting cycles with $n$ spikes per burst and cycles with $n+1$ spikes per burst is continuous, as can be observed in Fig. 6 (a). This is mainly due to the geometry of the critical manifold, which is cubicshaped, and therefore allows the true canard to extend all through the (central) repelling sheet $S_{1}^{r}$ of the critical manifold and to connect to a second repelling sheet $S_{2}^{r}$ along the fold curve $F^{-}$. However, the final spike-adding (in this case the fifth canard explosion) terminates due to a homoclinic bifurcation of the full system. The maximum number of spikes that the model may add upon parameter variations depends on other system parameters, in particular those affecting the timescales.

\section{The BRC model for parabolic bursting}

This section focuses on the BRC model [1], which is a phenomenological version of Plant model with two slow variables and one fast phase variable. We show that this model also possesses a folded-saddle singularity that organises the spike-adding mechanism, however via a discontinuous process in parameter space. The model is three-dimensional and given as follows:

$$
\begin{aligned}
\theta^{\prime} & =1-\cos \theta+A(x, y), \\
x^{\prime} & =\varepsilon_{x}\left(x_{\infty}(\theta)-x\right), \\
y^{\prime} & =\varepsilon_{y}\left(y_{\infty}(\theta)-y\right),
\end{aligned}
$$


(a)

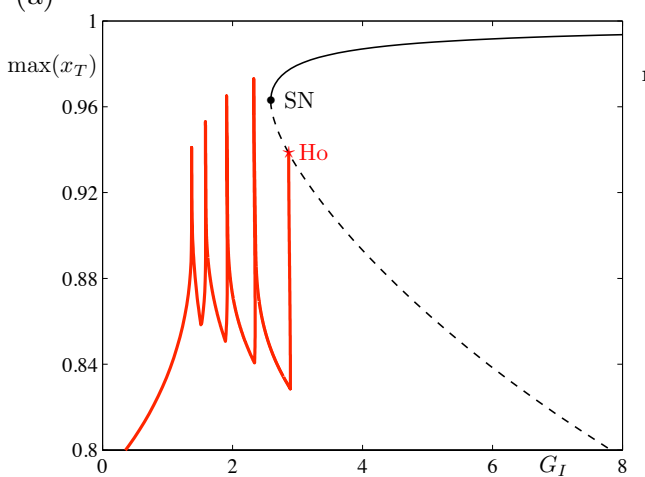

(b)

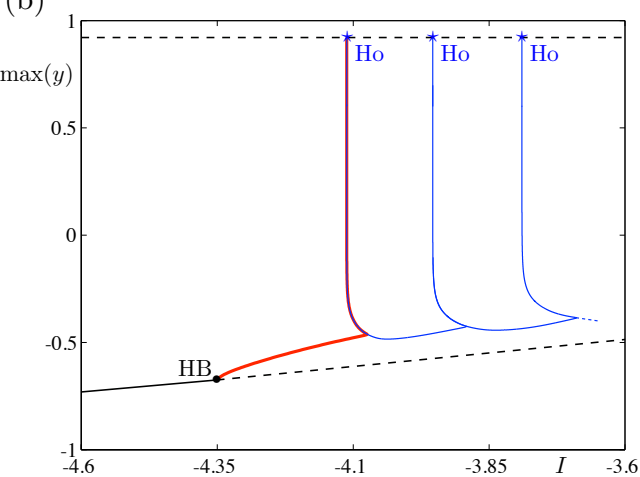

Figure 6: Comparison between the spike-adding mechanism in the Plant model (a) and in the BRC model (b). The adding is continuous (in parameter space) in the former and discontinuous in the latter. Solutions branches shown are branches of equilibria (black), branches of limit cycles born at a Hopf bifurcation labelled HB (red), and branches of limit cycles whose onset and offset correspond to a homoclinic bifurcation labelled Ho (blue, panel (b)). Stable (resp. unstable) equilibrium branches are traced in solid (resp. dashed) lines.

where $A(x, y)=\tanh (a x-b y+I)$ and $s_{\infty}(\theta)=\sin \left(p_{s}+\theta\right)$ for $s=x, y$. The quantities $a, b, I$ are parameters, and $\varepsilon_{s}(s=x, y)$ are small positive constants. The variable $\theta$ is a phase variable, therefore the phase space of system (18) is $\mathbb{S}^{1} \times \mathbb{R}^{2}$. To facilitate a slow-fast analysis of system (18), we henceforth write: $\varepsilon_{y}=\varepsilon$ and $\varepsilon_{x}=k \varepsilon_{y}=k \varepsilon$. Thus we will study the following slow-fast system with one fast variable and two slow variables

$$
\begin{aligned}
\theta^{\prime} & =1-\cos \theta+A(x, y), \\
x^{\prime} & =\varepsilon k\left(x_{\infty}(\theta)-x\right), \\
y^{\prime} & =\varepsilon\left(y_{\infty}(\theta)-y\right) .
\end{aligned}
$$

Changing to the fast time yields the following equivalent parametrisation of system (19)

$$
\begin{aligned}
\varepsilon \dot{\theta} & =1-\cos \theta+A(x, y)=f(\theta, x, y), \\
\dot{x} & =k\left(x_{\infty}(\theta)-x\right), \\
\dot{y} & =\left(y_{\infty}(\theta)-y\right) .
\end{aligned}
$$

The critical manifold $S^{0}$ of system (20) is given by

$$
S^{0}:=\{\cos \theta=1+A(x, y)\} .
$$

In what follows we will show that the critical manifold $S^{0}$, given by equation (21), possesses a folded-saddle singularity and that geometrically the repelling part of $S^{0}$ extends up to infinity thereby inducing spike-adding via a discontinuous process. To begin with, note that $S^{0}$ defines a $2 \pi$-periodic surface, with fold curve $F$ defined by $\left\{f_{\theta}=\sin \theta=0\right\}$, that is, corresponding to $\theta=0 \bmod 2 \pi$; see Fig. 7 for a phase-space representation of the critical manifold. Indeed, for $\theta \in(\pi / 2,3 \pi / 2) \bmod 2 \pi$, the equation defining the critical manifold is not satisfied since $|A(x, y)|<1$. Therefore, the fold curve $F$ is simply $\{\theta=0 \bmod 2 \pi\}$, which is equivalent to $A(x, y)=0$ in the $(x, y)$-plane of 
(a)

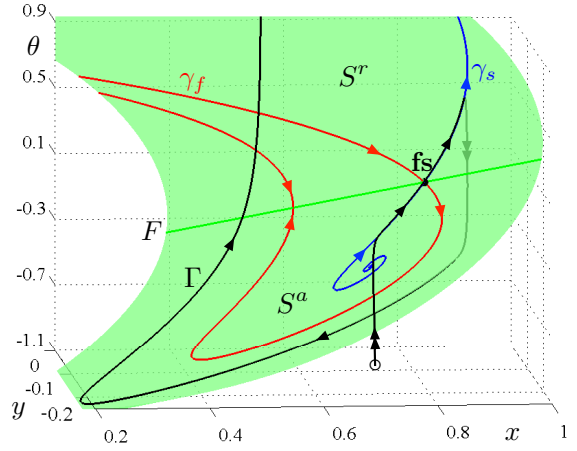

(b)

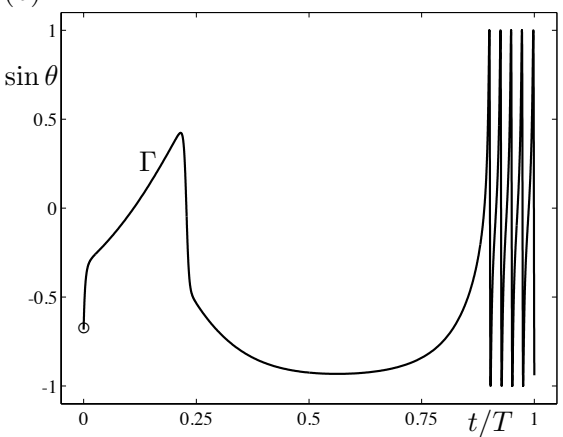

Figure 7: Solution of the BRC model (20) containing a canard segment. Panel (a) shows the trajectory in the phase space together with the critical manifold $S^{0}$ (attracting sheet $S^{a}$ and repelling sheet $S^{r}$, the fold curve $F$, the folded saddle (black dot) and its (un)stable manifolds (singular true canard $\gamma_{s}$ and singular faux canard $\gamma_{f}$ ). Panel (b) shows the time profile of the same trajectory, the plotted output being $\sin \theta$. In each panel, the circle denotes the initial condition.

slow variables, that is, the straight line of equation: $a x-b y+I=0$. It follows from slow-fast theory that systems with two slow variables and one fast variable, with a folded critical manifold, can display canard solutions; see $[9,5,58]$. In this context, canards correspond to connections between two-dimensional attracting and repelling slow manifolds, which are possible when folded singularities are present on the fold curve of the critical manifold. We now show that $S^{0}$ possesses a folded-saddle singularity. Taking the singular limit $(\varepsilon=0)$ of system (20) results in a DAE whereby the dynamics of the slow variables $x$ and $y$ are now constrained by an algebraic equation that corresponds to the critical manifold. Differentiate the constraints with respect to time and keeping on slow equation gives

$$
\begin{aligned}
-f_{\theta} \dot{\theta} & =f_{x} \dot{x}+f_{y} \dot{y}, \\
\dot{x} & =k\left(x_{\infty}(\theta)-x\right) .
\end{aligned}
$$

Rescaling time by a factor $-f_{\theta}$ in $(22)$ yields the DRS

$$
\begin{aligned}
\theta^{\prime} & =f_{x} \dot{x}+f_{y} \dot{y}, \\
x^{\prime} & =-f_{\theta} k\left(x_{\infty}(\theta)-x\right),
\end{aligned}
$$

where $y$ is replaced by its expression as a function of $\theta$ and $x$ on the critical manifold. The conditions to have a folded singularity in system (20) are therefore expressed by:

$$
\begin{aligned}
f_{\theta} & =0 \\
A_{x}(x, y) k\left(x_{\infty}(\theta)-x\right)+A_{y}(x, y)\left(y_{\infty}(\theta)-y\right) & =0,
\end{aligned}
$$

with $A_{x}(x, y)=a\left(1-A^{2}(x, y)\right)$ and $A_{y}(x, y)=-b\left(1-A^{2}(x, y)\right)$. The first equation of (24) forces the equilibrium of the DRS to be on the fold curve $F$ of $S^{0}$, that is, for $\theta=0 \bmod 2 \pi$, where the term $A(x, y)$ vanishes. Hence, the second equation of (24) reduces to

$$
a k\left(x_{\infty}(\theta)-x\right)-b\left(y_{\infty}(\theta)-y\right)=0,
$$


(a)

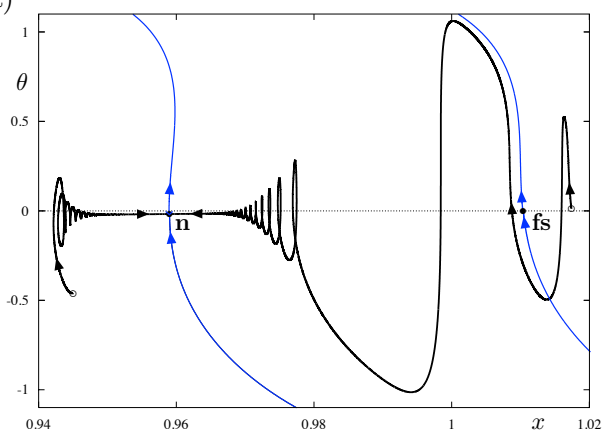

(b)

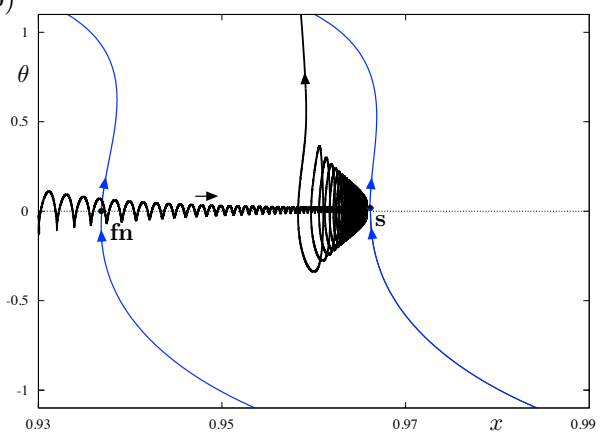

Figure 8: Solutions of the BRC theta model (20) corresponding to case 2, projected onto the $(x, \theta)$ plane and superimposed on top of the invariant manifolds of the two equilibria of the DRS for the same value of $I$. Panel (a): $I=-0.05$, the folded equilibrium (right) is a folded saddle fs and the true equilibrium is a node $\mathbf{n}$, which attracts the dynamics of the full system. Panel (b): $I=0.05$, the situation is opposite and the oscillatory pattern of the full dynamics is shaped by the passage through the folded node fn and near the true saddle s. In both panels, the arrows indicate the direction of the flow.

with: $y=(a x+I) / b$. Therefore, the coordinates of the folded singularities of system (20) are

$$
\begin{aligned}
& \theta_{\mathbf{f s}}=0 \bmod 2 \pi \\
& x_{\mathbf{f s}}=\frac{a k \sin p_{x}-b \sin p_{y}+I}{a(k-1)}, \\
& y_{\mathbf{f s}}=\frac{a x_{\mathbf{f s}}+I}{b} .
\end{aligned}
$$

The Jacobian matrix at the folded singularity $\left(\theta_{\mathbf{f s}}, x_{\mathbf{f s}}, y_{\mathbf{f s}}\right)$ has the following form

$$
\mathrm{J}_{\mathrm{fs}}=\left(\begin{array}{cc}
a k \cos p_{x}-b \cos p_{y} & -a k \\
-k\left(\sin p_{x}-x_{\mathbf{f s}}\right) & 0
\end{array}\right),
$$

Therefore, the condition for the folded equilibrium to be a saddle of the DRS is

$$
a k^{2}\left(\sin p_{x}-x_{\mathbf{f s}}\right)>0,
$$

which, according to the expression of $x_{\mathbf{f s}}$ in $(26)$, gives

$$
\frac{k^{2}}{|k-1|}\left(b \sin p_{y}-a \sin p_{x}-I\right)>0,
$$

that is,

$$
I<b \sin p_{y}-a \sin p_{x}
$$

It is not difficult to check that for parameter values typically used in system (18) (see for instance [49]), the folded singularity $\mathbf{f s}:=\left(\theta_{\mathbf{f s}}, x_{\mathbf{f s}}, y_{\mathbf{f s}}\right)$ given by (26) is a folded saddle. An illustration of the singular true canard, $\gamma_{s}$, flowing along the critical manifold $S^{0}$ through the folded saddle, is shown in Fig. 7 (a). 
Away from the singular limit, Fig. 7 (a) also shows a trajectory $\Gamma$, solution of the full system (20) with initial condition indicated by a circle, which closely follows the singular true canard. That is, in accordance to Fenichel theory [18], the solution is attracted and flows $\left(O(\varepsilon)\right.$-close) along the attracting sheet $S^{a}$ of the critical manifold. Subsequently it passes near $\mathbf{f}_{\mathbf{s}}$, where Fenichel theory does not apply anymore, and then continues close to the repelling side $S^{r}$ of the critical manifold. Then it jumps (back to the attracting side) and finally flows until it is ejected when reaching the fold curve at a jump point. The same orbit segment of the full system is shown in the time domain in Fig. 7 (b).

Returning back to the geometry of the ( $2 \pi$-periodic) critical manifold $S^{0}$, we note that every connected component of the manifold has a single fold and consequently both the repelling and the attracting sheet of the critical manifold extend to infinity; see Fig. 7 (a). It turns out that the single fold curve corresponds here as well to a curve of SNIC points of the fast subsystem; this is a clear trademark of all parabolic bursters. Therefore the repelling segment of the singular true canard extends to infinity and the spike-adding process is discontinuous in parameter space; see Fig. 6 (b) for an illustration of this discontinuous spike-adding bifurcation scenario. Specifically, each branch of limit cycles with a given number of spikes per burst terminates at a homoclinic bifurcation, and all but the first one (born at a Hopf bifurcation) also stems from a homoclinic bifurcation. This is in contrast to the Plant model, where the solution branch of limit cycles gaining more and more spikes as a parameter is varied, is continuous; see Fig. 6 (a) for an illustration of this continuous spike-adding bifurcation scenario. This difference emerges due to the finite length of the repelling sheet of the critical manifold - and also of the singular true canard - and the fact that this repelling sheet connects two attracting sheets.

Finally, note that the DRS of system (20) has an additional branch of equilibria, which are equilibria of the full system. In [49], two cases were considered depending of the relative size of $\varepsilon_{x}$ with respect to $\varepsilon_{y}$ : case 1 when $k=\frac{\varepsilon_{x}}{\varepsilon_{y}} \gg 1$ and case 2 when $k \ll 1$. In each case, varying parameter $I$ allows to move the system across the bursting regime; see figures 8 and 9 of [49]. The relation (30) is independent of $k$, therefore in either case (1 and 2) by varying $I$ one observes the same bifurcations of the underlying structure of folded singularities within the system. In particular, varying parameter $I$ changes the type of folded singularity from one type to the other via a so-called folded-saddle node type II (FSNII), which occurs when the branch of folded singularities intersects the branch of true singularities of the system; see [34] for more details about this scenario. The condition for FSN II in system (7) is given as follows:

$$
I=b \sin p_{y}-a \sin p_{x} .
$$

The main difference between the two cases is the type of folded singularity that organises the dynamics in the bursting regime. In case 1 (figure 8 of [49]), the bursting is initiated when the limit cycle of the slow subsystem collides with the fold curve of the critical manifold, making a folded homoclinic connection with the folded saddle. The burst then terminates when $I$ satisfies equation (31). An interesting topic for future work will be to investigate more closely the transition from bursting to spiking in this case, which may be related to the torus canard phenomenon [6,31]. In contrast, case 2 sees the burst initiate through the FSN II transition and terminates with the disappearance of an 
unstable limit cycle of the average system studied in [49]; see Fig. 8. In both cases, the FSN II corresponds to one end of the bursting regime. Note that folded nodes can also appear in the Plant model, however the set of parameters have to be adjusted away from the typical parameters considered by Plant; we do not present this parameter regime here. A follow-up study will analyse more closely the transition from the folded-saddle to the folded-node regime, via the folded-saddle node bifurcation, in link with a change of type of homoclinic bifurcation (presumably, from SNIC to saddle-homoclinic bifurcation via saddlenode homoclinic bifurcation).

\section{Atoll model from Ermentrout and Kopell}

Finally, we consider the theta model by Ermentrout and Kopell [16, 30] in its simplest form, also known as the Atoll model; see [25, 28]. In this section we will show that the model also admits canard solutions that organizes spike-adding in the bursting regime, which does not seem to have been reported before, even though canards on the torus have been studied from a theoretical perspective in $[23,53,54,55]$. This canonical model (also called theta model) is a twodimensional phase reduction of parabolic bursters (i.e. with the two-torus $\mathbb{T}^{2}$ as phase space) and is given as follows:

$$
\begin{aligned}
& \phi^{\prime}=1-\cos \phi+(1+\cos \phi) \cos \theta, \\
& \theta^{\prime}=\varepsilon,
\end{aligned}
$$

where $(\phi, \theta) \in \mathbb{T}^{2}$ and $\varepsilon>0$ is the small parameter. The usual rescaling brings the slow-time system

$$
\begin{aligned}
\varepsilon \dot{\phi} & =1-\cos \phi+(1+\cos \phi) \cos \theta, \\
\dot{\theta} & =1 .
\end{aligned}
$$

System (33) is a slow-fast dynamical system with one fast variable and one slow variable. Its critical manifold is given by

$$
\cos \theta=\frac{\cos \phi-1}{\cos \phi+1}
$$

Following the same procedure as in the previous sections, we find fold points at $(\phi, \theta)=\left(0 \bmod 2 \pi, \frac{\pi}{2} \bmod \pi\right)$. Therefore the critical manifold $S^{0}$ of system $(33)$ is formed by a curve traced on $\mathbb{T}^{2}$, and with two fold points. It is easy to see that both fold points are jump points because the normal switching conditions (see previous sections) are satisfied at both points. Note that a folded saddle is not possible here due to the reduced dimension of the system. However, canard solutions are still possible, namely so-called jump-on canards or transitory canards [8]. These canards have been studied in various papers including [13] where they were termed canards with jump (see figure 4(d) in [13]). Following the approach introduced in [12] (for periodically-forced slow-fast systems), we plot both the solution of the Atoll model super-imposed with its critical manifold $S^{0}$ in the time-domain; see Fig. 9. Every component of the critical manifold resembles an atoll (see $[25,28]$ ), hence the name "Atoll model". In the neuronal context, the quiescent phase of the bursting solution (as visualised in time) 
(a)

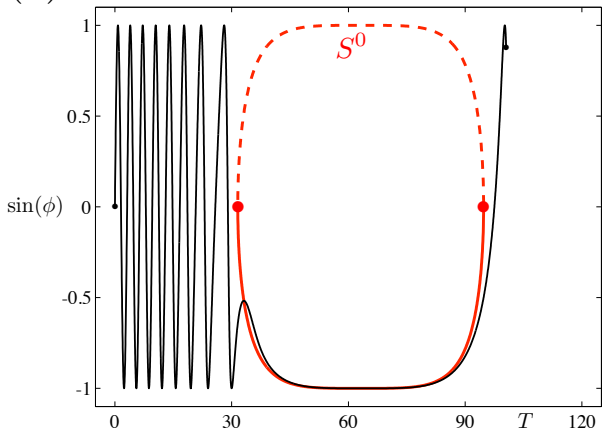

(c)

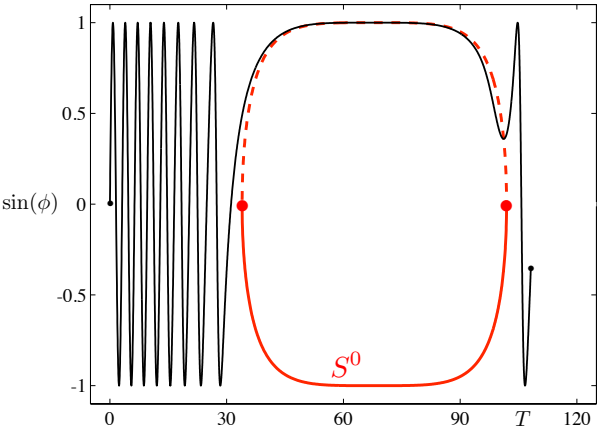

(e)

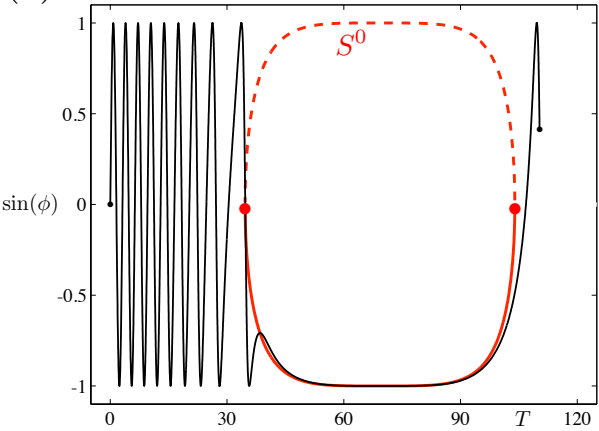

(b)

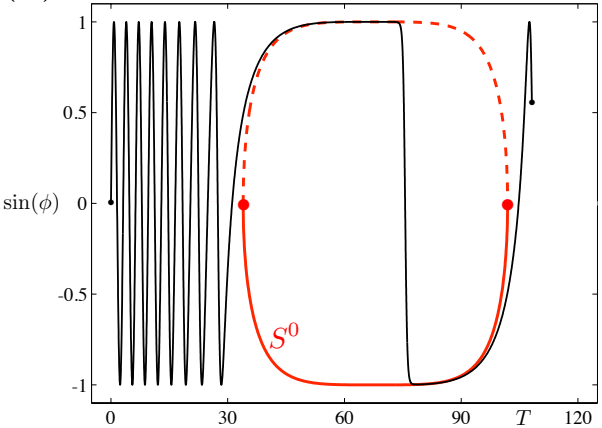

(d)

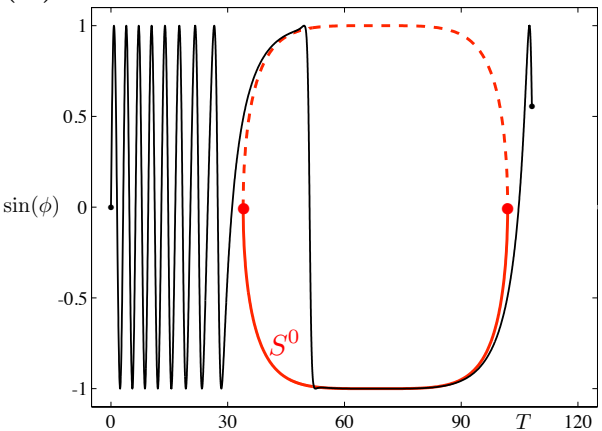

(f)

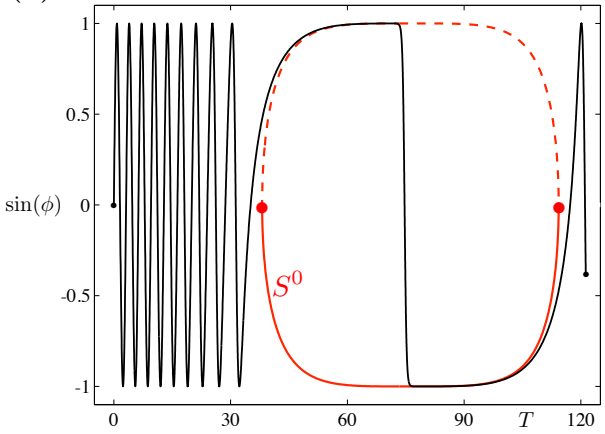

Figure 9: Segments of bursting solutions of the Atoll model (32) along a canard-mediated spike-adding transition obtained by varying parameter $\varepsilon$. In each panel, the time profile of the orbit segment in shown together with the critical manifold $S^{0}$ (attracting part in solid lines, repelling part in dashed lines) and its two fold points (red dots). These orbit segments are part of limit cycles and they have been computed by numerical continuation, solving a one-parameter family of two-point boundary-value problems. The boundary points are shown as black dots in each panel. The associated solution branch is shown in Fig. 10.

corresponds to the segments of trajectory that are slow and follow the bottom part of the Atoll-shaped critical manifold. The novelty here (from the neuronal dynamics viewpoint only) is that during the fast parts of the trajectory, which correspond to groups of spikes (i.e. the bursts), the number of spikes can vary (upon parameter variation) and the transition orbits are canards. During these 
(a)

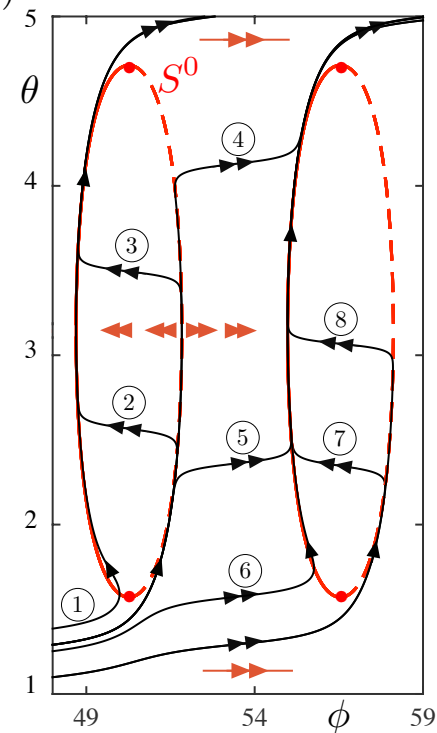

(b)

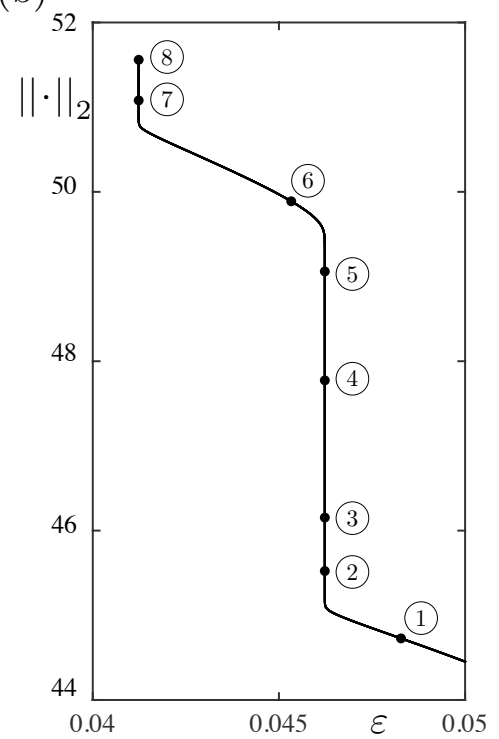

Figure 10: Spike-adding canard transitions in the Atoll model (32) computed by the continuation of orbit segments solution to boundary-value problems (see, e.g., [9] for details on this numerical approach). Panel (a) shows eight orbit segments in the phase plane $(\phi, \theta)$, the direction of time along each segment being indicated by black arrows; the red curves represent two copies of the ( $2 \pi$-periodic) critical manifold $S^{0}$ to better highlight the canard dynamics. We also show the fold points (red dots) of $S^{0}$, along which attracting (resp. repelling) sheets are shown as red solid (resp. dashed) lines, as the fast flow (red) double arrows indicates. Panel (b) shows the computed solution branch, for varying $\varepsilon$, with the eight particular solutions labelled 1 to 8 and indicated by a black dot on the branch. Each quasi-vertical segment of the computed branch corresponds to a spike-adding transition through canards, therefore the solutions 2 to 5,7 and 8 contain canard segments.

fast transitions the trajectories visit the upper part of the Atoll-shaped critical manifold when looking at the time profile; see the transition orbits 2 to 5,7 and 8 in Fig. 9. Indeed the jump-on canards organise the transition from $n$ to $n+1$ spikes. In the phase plane (see Fig. 10 (a)), the part of the Atoll where typical dynamics occurs is the left part of the Atoll, and the canard dynamics takes place on the right part. Note that the orbits 1, 3, 5, 6 and 8 of Fig. 10 correspond to panels (a), (b), (d), (e) and (f) of Fig. 9, respectively. To conclude, the spike-adding mechanism of the Atoll model is still canard-induced but different (in terms of the slow flow) to the higher-dimensional parabolic bursters that we have investigated. To this extent, a matter of future research (which goes beyond the scope of the present paper) will be to investigate if the phase variables are not masking the true nature of the spike-adding mechanism. That is, it will interesting to convert the slow dynamics of the Atoll model to cartesian coordinates (via an appropriate coordinate change), therefore recovering a 4D system, and subsequently compare with the slow flow to that of a system with a folded-saddle singularity. 


\section{Discussion and future work}

In this work, we have studied for the first time parabolic bursting from the perspective of canards. Employing singular perturbation theory in tandem with numerical continuation adapted for slow-fast systems [9] reveals that new spikes emerge during parabolic bursting as a result of a passage through foldedsaddle canards. We also bring out the existence of a new bifurcation which we term folded homoclinic bifurcation, corresponding to a homoclinic bifurcation of the desingularised reduced system involving the folded-saddle singularity. This global bifurcation of the desingularised slow flow is a key element allowing the transition to solutions with one spike from purely quiescent oscillations (slow waves) as a first step leading to the parabolic bursting regime. This phenomenon persists across biophysical models and also three-dimensional phase models that exhibit at least two slow variables. This has lead us towards the construction of a canonical four-dimensional polynomial model (based on a modified Hindmarsh-Rose system), which to date provides the best parsimonious mathematical approximation of the Plant model and, therefore, constitutes a prototypical phenomenological parabolic burster. We have also seen that in planar phase models of parabolic bursters, like the Atoll model, the spike-adding mechanism still involves canard solutions, but of different type, namely jump-on canards.

Canard-induced spike-adding phenomena are interesting beyond the simple case of parabolic bursting, and our working hypothesis is that it should be a persistent mechanism across all main classes of bursters, namely parabolic, squarewave and elliptic bursters. Canard-induced spike-adding transitions have been reported and investigated in various contexts, both in transient and asymptotic dynamics, mostly in the case of square-wave bursters [24, 43, 59], even though a complete theory for such phenomena is still lacking. Our recent work demonstrates that in square wave bursters spike-adding transitions occur through multiple canard explosions [11]. Interestingly, studies have also shown the existence of canards in elliptic bursters [27], however it remains to be investigated if elliptic bursters display a spike-adding process via canards. Verifying our hypothesis first in elliptic bursters and possibly in further classes of bursters is a matter of future considerations, which we hope will lead to a general classification of spikeadding mechanisms. Our on-going work is also concerned with the unfolding of these bifurcation scenarios. For example, we have recently investigated the passage through a folded-node singularity in an extended version of a square-wave burster. This extended model displayed a mix between Mixed-Mode Oscillations and bursting oscillations [11]. Therein, we highlighted the importance of folded singularities, and associated canard solutions, in bursting models with two slow variables and two fast variables. In this context, a complete understanding of the transition from folded saddle to folded node (via folded saddle-node bifurcation) is a natural future research direction. In particular, it will be interesting to relate that transition of folded singularities with a possible change in the type of homoclinic bifurcation (from SNIC to saddle-homoclinic) occurring in the bursting system under consideration. 


\section{References}

[1] S. M. Baer, J. Rinzel, H. Carillo, Analysis of an autonomous phase model for neuronal parabolic bursting, J. Math. Biol. 33(3) (1995) 309-333.

[2] E. Benoît, Canards et enlacements, Publ. Math. IHÉS 72(1) (1990) 63-91.

[3] R. Bertram, M. J. Butte, T. Kiemel, A. Sherman, Topological and phenomenological classification of bursting oscillations. Bulletin of mathematical biology 57(3): 413-439, 1995.

[4] W. Bialek, F. Rieke, R.D.R. van Steveninck and D. Warland, (1999). Spikes: Exploring the Neural Code (Computational Neuroscience).

[5] M. Brøns, M. Krupa and M. Wechselberger, Mixed-mode oscillations due to the generalized canard mechanism, Fields Institute Communications 49 (2006) 39-63.

[6] J. Burke, M. Desroches, A. M. Barry, T. J. Kaper and M. A. Kramer, A showcase of torus canards in neuronal bursters, The Journal of Mathematical Neuroscience, 2(3) (2012).

[7] J.-L. Callot, Solutions visibles de l'équation de Schrödinger, In: Mathématiques finitaires et analyse non standard, Tome 1., Publi. Math. de l'Univ. Paris VII nº 31 (1985) 105-119.

[8] P. De Maesschalck, F. Dumortier, R. Roussarie, Canard-cycle transition at a fast-fast passage through a jump point, C. R. Acad. Sci. Math. 352(1) (2014) 27-30.

[9] M. Desroches, J. Guckenheimer, B. Krauskopf, C. Kuehn, H. M. Osinga and M. Wechselberger, Mixed-mode oscillations with multiple time scales, SIAM Review 54(2) (2012) 211-288.

[10] M. Desroches, M. Krupa and S. Rodrigues, Inflection, canards and excitability threshold in neuronal models, J. Math. Biol. 67(4) (2013) 9891017.

[11] M. Desroches, T. J. Kaper, M. Krupa, Mixed-mode bursting oscillations: Dynamics created by a slow passage through spike-adding canard explosion in a square-wave burster, Chaos 23(4) (2013) 046106.

[12] M. Diener, Nessie et les canards, IRMA preprint (1979).

[13] M. Diener, The canard unchained or how fast/slow dynamical systems bifurcate, The Mathematical Intelligencer, 6(3) (1984) 38-49.

[14] E. J. Doedel, A. R. Champneys, F. Dercole, T. F. Fairgrieve, Yu A. Kuznetsov, B. Oldeman, R. C. Paffenroth, B. Sandstede, X. J. Wang and C. H. Zhang, AUTO-0\%p: Continuation and bifurcation software for ordinary differential equations, 2007. Available at: http://indy.cs.concordia.ca/auto/.

[15] F. Dumortier and R. Roussarie, Canard cycles and center manifolds, Mem. Amer. Math. Soc. 121(577) (1996). 
[16] G. B. Ermentrout, N. J. Kopell, Parabolic bursting in an excitable system coupled with a slow oscillation, SIAM J. Appl. Math. 46(2) (1986) 233-253.

[17] G. B. Ermentrout, Simulating, analyzing, and animating dynamical systems: a guide to XPPAUT for researchers and students, SIAM (2002).

[18] N. Fenichel, Geometric singular perturbation theory for ordinary differential equations, J. Differential Equations 31(1) (1979) 53-98.

[19] R. FitzHugh, Mathematical models of threshold phenomena in the nerve membrane. Bull. Math. Biophysics, 17 (1955) 257-278

[20] A. Ghosh, D. Roy, V. K. Jirsa, Simple model for bursting dynamics of neurons, Phys. Rev. E 80(4) (2009) 041930.

[21] M. Golubitsky, K. Josic, T. J. Kaper, An unfolding theory approach to bursting in fast-slow systems, In: Global Analysis of Dynamical Systems: Festschrift dedicated to Floris Takens on the occasion of his 60th birthday, H. W. Broer, B. Krauskopf, G. Vegter (Eds.), Institute of Physics Publ., 2001, pp. 277-308.

[22] W. Govaerts, A. Dhooge, Bifurcation, bursting and spike generation in a neural model. Int. J. Bifurcation and Chaos 12(8) (2002) 1731-1741.

[23] J. Guckenheimer, Y. Ilyashenko, The duck and the devil: canards on the staircase, Moscow Math. J 1(1) (2001) 27-47.

[24] J. Guckenheimer and C. Kuehn, Computing slow manifolds of saddle type, SIAM J. Appl. Dyn. Syst. 8(3) (2009) 854-879.

[25] F. C. Hoppensteadt, E. M. Izhikevich, Weakly connected neural networks, Appl. Math. Sci. Vol. 126, Springer (1997).

[26] J. L. Hindmarsh and R.M. Rose, A model of neuronal bursting using three coupled first order differential equations. Proc. R. Soc. London, Ser. B (1984) 221:87-102.

[27] E. M. Izhikevich, Neural excitability, bursting and spiking Int. J. Bifurcation and Chaos 10(6) (2000) 1171-1266.

[28] E. M. Izhikevich, Dynamical systems in neuroscience: the geometry of excitability, MIT press (2007).

[29] C. K. R. T. Jones, Geometric singular perturbation theory, In: "Dynamical Systems", R. Johnson ed., Lecture Notes in Mathematics vol.1609, pp. 44-118, Springer, Berlin, 1995.

[30] N. J. Kopell, and G. B. Ermentrout, Subcellular oscillations and bursting, Math. Biosci. 78(2) (1986) 265-291.

[31] M. A. Kramer, R. D. Traub and N. J. Kopell, New dynamics in cerebellar purkinje cells: torus canards, Phys. Rev. Lett. 101(6) (2008) 068103.

[32] M. Krupa, and P. Szmolyan, Extending geometric singular perturbation theory to nonhyperbolic points - fold and canard points in two dimensions, SIAM J. Math. Anal. 33(2): 286-314, 2001. 
[33] M. Krupa and P. Szmolyan, Relaxation oscillation and canard explosion, J. Differential Equations 174(2) (2001) 312-368.

[34] M. Krupa and M. Wechselberger, Local analysis near a folded saddle-node singularity, J. Differential Equations 248(12) (2010) 2841-2888.

[35] Y. A. Kuznetsov, Elements of applied bifurcation theory, Third Edition, Springer, 2004.

[36] Wolfram Research, Inc., Mathematica, Version 10.0, Champaign, IL (2014).

[37] A. Milik, P. Szmolyan, H. Loeffelmann and E. Groeller, Geometry of mixedmode oscillations in the $3 d$ autocatalator, Int. J. Bifurcation and Chaos 8(3) (1998) 505-519.

[38] E. F. Mishchenko and N. K. Rozov, Differential equations with small parameters and relaxation oscillations, Plenum Press (New York) (1980).

[39] E. F. Mishchenko, Y. S. Kolesov, A. Y. Kolesov and N. K. Rozov, Asymptotic methods in singularly perturbed systems, Consultants Bureau (New York) (1994).

[40] J. Mitry, M. McCarthy, N. J. Kopell and M. Wechselberger, Excitable neurons, firing threshold manifolds and canards, J. Math. Neurosci. 3 (2013) 12 .

[41] J. Mitry and M. Wechselberger, Folded saddles \& faux canards, submitted preprint, 2015.

[42] J. Nagumo, S. Arimoto and S. Yoshizawa, An active pulse transmission line simulating nerve axon. Proc IRE. 50 (1962) 2061-2070.

[43] J. Nowacki, H. M. Osinga and K. Tsaneva-Atanasova, Dynamical systems analysis of spike-adding mechanisms in transient bursts, The Journal of Mathematical Neuroscience 2(1) (2012) 1-28.

[44] R. E. Plant, M. Kim, On the mechanism underlying bursting in the Aplysia abdominal ganglion R15 cell, Math. Biosci. 26 (3) (1975) 357-375.

[45] R. E. Plant, M. Kim, Mathematical description of a bursting pacemaker neuron by a modification of the Hodgkin-Huxley equations, Biophys. J. 16 (3) (1976) 227-244.

[46] R. E. Plant, The effects of calcium $^{++}$on bursting neurons: A modelling study, Biophys. J. 21(3) (1978) 217-237.

[47] R. E. Plant, Bifurcation and resonance in a model for bursting nerve cells, J. Math. Biol. 11(1) (1981) 15-32.

[48] J. Rinzel, A formal classification of bursting mechanisms in excitable systems, In: Proceedings of the International Congress of Mathematicians, Vol. 1, 1986, pp. 1578-1593.

[49] J. Rinzel and Y. S. Lee, Dissection of a model of neuronal parabolic bursting, J. Math. Biol. 25(6) (1987) 653-675. 
[50] J. Rinzel and G. B. Ermentrout, Analysis of neural excitability and oscillations, In: Methods in Neuronal Modeling, From ions to networks, second edition, C. Koch and I. Segev eds., pp. 251-293, The MIT Press, Cambridge, MA, 1998

[51] P. Roper, P. C. Bressloff and A. Longtin, A phase model of temperaturedependent mammalian cold receptors, Neural Comput. 12(5) (2000) 10671093.

[52] I. Samengo, G. Mato, D.H. Elijah, S. Schreiber and M.A. Montemurro, Linking dynamical and functional properties of intrinsically bursting neurons, J. Comput. Neurosci 35(2) (2013) 213-230.

[53] I. V. Schurov, Ducks on the torus: existence and uniqueness, J. Dyn. Control Syst. 16(2) (2010) 267-300.

[54] I. V. Schurov, Canard cycles in generic fast-slow systems on the torus, Trans. Moscow Math. Soc. 71 (2010) 175-207.

[55] I. V. Schurov, N. Solodovnikov, Duck factory on the two-torus: multiple canard cycles without geometric constraints, arXiv:1405.3251 (2014).

[56] P. Smolen, D. Terman and J. Rinzel, Properties of a bursting model with two slow inhibitory variables, SIAM J. Appl. Math. 53(3) (1993) 861-892.

[57] C. Soto-Treviño, N. Kopell and D. Watson, Parabolic bursting revisited, J. Math. Biol. 35(1) (1996) 114-128.

[58] P. Szmolyan and M. Wechselberger, Canards in $\mathbb{R}^{3}$, J. Differential Equations $177(2)$ (2001) 419-453.

[59] D. Terman, Chaotic spikes arising from a model of bursting in excitable membranes, SIAM J. Appl. Math. 51(5) (1991) 1418-1450. 